Review

\title{
Clinical strategies to manage adult glioblastoma patients without MGMT hypermethylation
}

\author{
Delin Liu, Tianrui Yang, Wenbin Ma, Yu Wang ${ }^{\bowtie}$ \\ Department of Neurosurgery, Peking Union Medical College Hospital, Beijing, China. \\ $\square$ Corresponding author: Yu Wang, M.D., E-Mail: ywang@pumch.cn; Tel.: +8615311860318; Address: Peking Union Medical College Hospital (East), No. 1 \\ Shuaifuyuan Wangfujing Dongcheng District, Beijing, China. \\ (1) The author(s). This is an open access article distributed under the terms of the Creative Commons Attribution License (https://creativecommons.org/licenses/by/4.0/).
} See http://ivyspring.com/terms for full terms and conditions.

Received: 2021.06.06; Accepted: 2021.11.21; Published: 2022.01.01

\begin{abstract}
Glioblastoma (GBM) is a highly malignant brain tumor with a dismal prognosis. Standard therapy for GBM comprises surgical resection, followed by radiotherapy plus concomitant and adjuvant temozolomide (TMZ) therapy. The methylation status of the O6-methylguanine DNA methyltransferase (MGMT) promoter is one of the most essential predictive biomarkers for patients with GBM treated with TMZ. Patients with an unmethylated MGMT promoter (umMGMT), who comprise $60 \%$ of patients with GBM, present an even worse prognosis because of TMZ resistance. Radiotherapy with various fractionation, chemotherapy compensating for TMZ, targeted therapy against diverse oncogenic pathways, immunotherapy of vaccine or immune checkpoint inhibitor, and tumor treating fields have been studied in umMGMT GBM patients. However, most efforts have yielded negative results or merely minimal improvements. Therefore, effective patient subgroup selection concerning precision medicine has become the focus. By assigning different treatments to the corresponding patient subgroups, a better curative effect and subsequently prolonged survival can be achieved. In this review, we re-evaluate the value of standard TMZ therapy and summarize the new clinical strategies and attempts to treat patients with umMGMT, which yielded positive and negative results, to provide alternative treatment options and discuss future directions of umMGMT GBM treatment.
\end{abstract}

Key words: glioblastoma, MGMT, methylation, therapy, clinical trial

\section{Introduction}

Glioblastoma (GBM) is the most common and aggressive primary malignant brain tumor in adults, accounting for $54 \%$ of all adult gliomas [1]. Despite optimized medical care, the median overall survival (OS) remains unsatisfactory. Standard therapy for newly diagnosed GBM includes surgical resection at maximum safety, followed by radiotherapy, as well as concomitant and adjuvant temozolomide (TMZ) therapy [2,3]; however, the population-based 5-year survival rate is still less than $10 \%[4,5]$.

TMZ is an alkylating agent that methylates the guanine base of DNA, inducing futile mismatch repair [6-8]. O6-methylguanine-DNA-methyltransferase (MGMT) removes the alkyl group, reversing the effect of TMZ. The expression of MGMT is silenced by MGMT promoter methylation [9-11]. Therefore, the unmethylated status of the MGMT promoter is one of the most important causes of TMZ resistance, leading to a significantly more dismal survival rate [2,3,12-14] (Figure 1a). A meta-analysis pooled the survival from five phase III clinical trials, suggesting the OS and progression free survival (PFS) of umMGMT were 14.11 months and 4.99 months, respectively [15]. Notably, MGMT is a reliable predictive biomarker for GBM in adults and the elderly; however, the value in children is less remarkable [16]. In the newly released $2021 \mathrm{WHO}$ classification of CNS tumors [17], glioblastoma lies in the adult diffuse glioma category, whereas pediatric gliomas are separately grouped with other important molecular markers such as H3K27-alteration and H3G34 mutation. Therefore, adult glioblastoma patients are mainly discussed in this review. 
$\mathbf{A}$

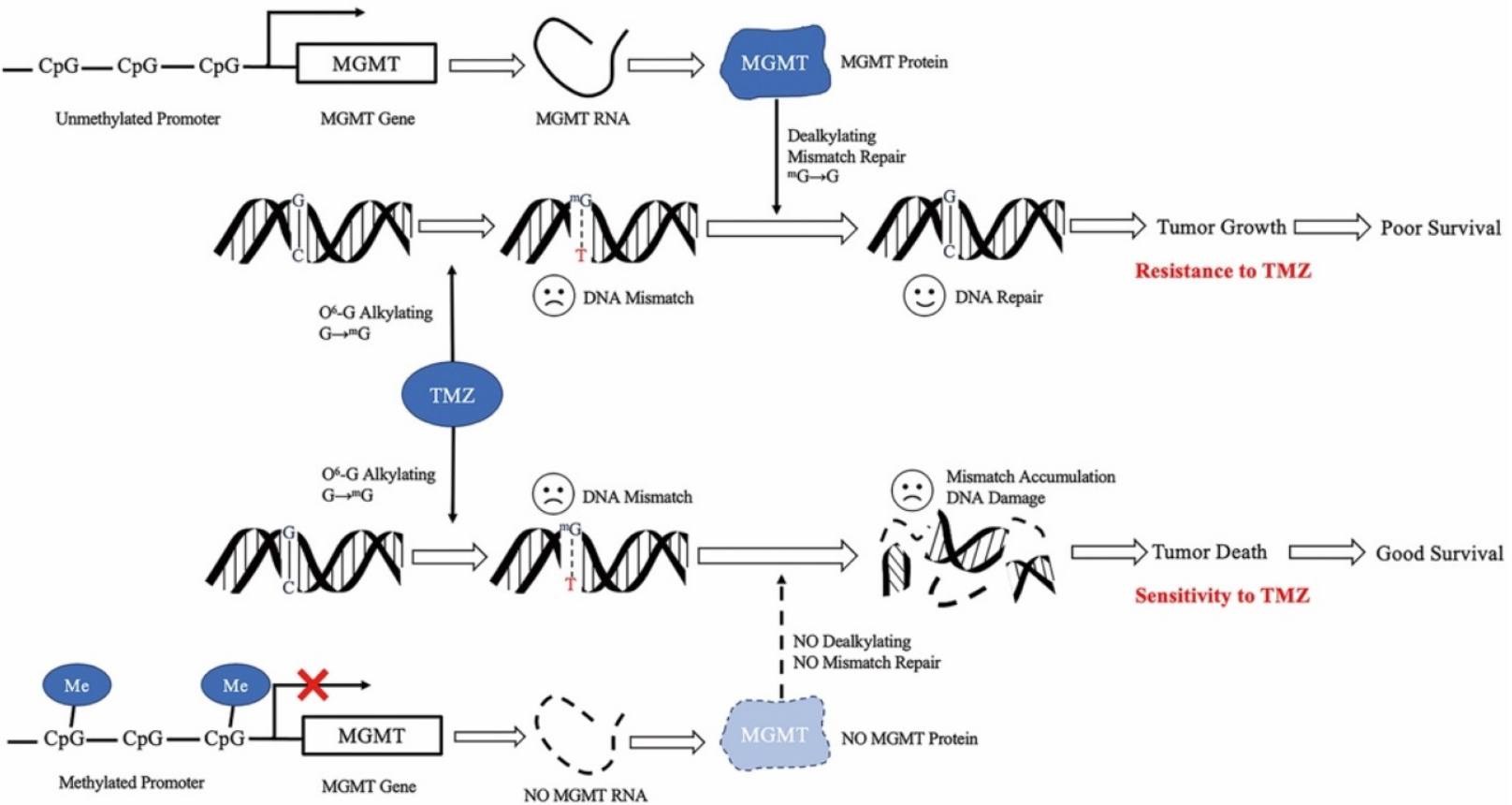

B

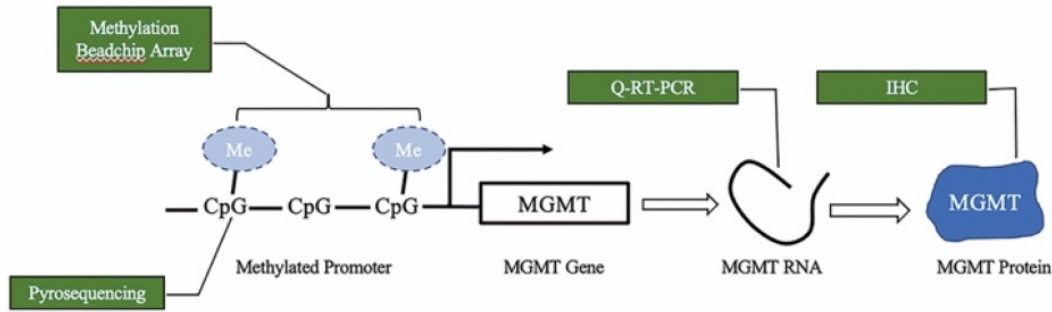

Figure 1. Mechanism of MGMT promoter methylation. a. Proposed role of MGMT promoter methylation as a predictive biomarker for TMZ. TMZ methylates the O6 position of guanine, leading to DNA mismatch. Unmethylated MGMT promotes TMZ resistance. When the MGMT promoter is unmethylated, transcription of the MGMT gene results in high MGMT protein expression, which is able to remove the alkylation adducts of $\mathrm{mG}$ and repair the mismatch, leading to tumor growth. When the MGMT promoter is methylated, silencing of transcription results in low level MGMT protein expression. Without dealkylation by MGMT protein, mismatch accumulates and causes DNA damage, leading to tumor death. b. Approaches to measure MGMT methylation status. Methylation BeadChip array analyzes the methylation spectrum of MGMT promoter. Pyrosequencing measures the average degree of methylation at the CPG site of MGMT promoter to determine the methylation status. Quantitative RT-PCR and immunochemistry analyze the level of MGMT RNA and protein, respectively. TMZ, temozolomide, MGMT O6-methylguanine DNA methyltransferase, mMe methyl, G guanine, mG O6-methylguanine, T thymine, Q-RT-PCR quantitative real-time PCR, IHC immunohistochemistry

Great debate over the use of TMZ in umMGMT patients has existed since the discovery of MGMT as a biomarker. In this review, we summarize clinical studies that aimed to treat patients with umMGMT GBM and reported both positive and negative outcomes, and discuss current treatment strategies and future treatment directions for patients with umMGMT GBM.

\section{Defining unmethylated MGMT}

To date, the MGMT status has been measured at the protein level using immunohistochemistry (IHC), RNA level using quantitative real-time PCR (Q-RT-PCR), promoter methylation level using pyrosequencing, or as methylation profiling with the Illumina BeadChip array[18] (Figure 1b).

The assessment of the MGMT status at the protein or RNA level was unable to predict survival. IHC has yielded inconsistent results $[19,20]$, because of observer variability and poor reproducibility. Q-RT-PCR that measures RNA levels has yielded similar results in differentiating the predictive value [19]. This limited discrimination is probably because of the mixed RNAs extracted from normal cells.

Pyrosequencing is currently considered the most reliable approach to quantitatively measure the methylation status $[20,21]$. Methylation of the MGMT promoter is not a qualitative parameter with only two modes. Instead, quantitative measurement is needed. A retrospective study in Norway analyzed 48 GBM patients, and the cutoff value with the best prognosis was set to $7 \%$ methylation of MGMT using pyrosequencing [21]. Another study in Italy set the cutoff similarly at 9\% [22]. Studies have also discovered a grey zone in between, which comprises approximately $10 \%$ of patients. Patients with hypermethylated and grey zone MGMT have better prognoses than those with umMGMT [23]. 
In recent years, studies have attempted to measure MGMT methylation in cell-free DNA (cfDNA) using methylation-specific PCR or pyrosequencing when the tumor tissue is not available. Although the results of pyrosequencing of cfDNA correlated with OS and PFS, both methods have low sensitivity and high negative predictive value [24]. Additionally, researchers have attempted to evaluate the MGMT methylation status using imaging parameters, such as the apparent diffusion coefficient $[25,26]$; however, these results have not been accepted to measure MGMT in clinical cases.

\section{Strategies to treat umMGMT patients}

\subsection{Surgery}

Surgical resection has been considered imperative for newly diagnosed GBM (nGBM) and possesses a certain value for recurrent GBM (rGBM). A greater extent of surgical resection of the contrast-enhancing tumor volume (CE-TV) is strongly associated with both prolonged OS and PFS [3,27-30]. Gross total resection (GTR) is associated with even longer OS (31 months) and PFS (6 months) than subtotal resection (STR) (OS 15 months, PFS 4 months) in umMGMT patients. A retrospective study reported that the extent of resection (EOA) beyond $70 \%$ and residual tumor volume (RTV) below $1.5 \mathrm{~cm}^{3}$ is already of better prognosis for umMGMT patients [31]. This result is stricter for mMGMT patients $(98 \%$ and $1 \mathrm{~cm}^{3}$, respectively). However, a study by Sales et al. focusing on umMGMT patients suggested that complete resection of the CE-TV did not result in improved survival [32]. Therefore, maximum safe resection, which avoids an aggressive surgical approach, is recommended instead of complete resection of important functional structures.

Laser Interstitial Thermal Therapy (LITT) is a new technique available as an alternative for deep, irresectable GBM patients, which utilizes the laser heat effect to destroy tumor cells with minimal invasion. LITT has been reported to annihilate deep-seated nGBM, rGBM, recurrent brain metastasis and radiation necrosis [33-35]. A cohort of 20 nGBM patient has reached OS of 36.2 months and PFS of 3.5 months after LITT operation [36]. For now, LITT works as a salvage treatment option only when open surgery is not applicable.

\subsection{Standard $T M Z$ radiochemotherapy}

The phase III EORTC-NCIC trial indicated that radiotherapy (60 Gy/30) plus concomitant (75 $\left.\mathrm{mg} / \mathrm{m}^{2} / \mathrm{d}\right)$ and adjuvant $\left(150-200 \mathrm{mg} / \mathrm{m}^{2}\right.$ for 5 days each 28-day cycle) TMZ therapy was able to improve OS and PFS for nGBM patients compared with radiotherapy alone. This treatment, known as the
Stupp's protocol, is now deemed the standard therapy for nGBM patients, especially for those with a methylated MGMT promoter. UmMGMT patients also benefit from radiotherapy plus TMZ, with a prolonged OS and PFS [12]. The major problems of the standard therapy are acquired TMZ resistance especially for umMGMT patients and intolerance of adverse effects due to TMZ or radiation, such as nausea, neutropenia, brain edema and radiation necrosis. For elderly patients who are not able to tolerate the intensiveness of standard radiochemotherapy, adjusted treatment plans are available. Hypofractionated radiation (40 Gy/15) plus TMZ was effective in a phase III clinical trial [37]. If even hypofractionated radiochemotherapy is intolerable, umMGMT GBM patients should choose radiotherapy alone over TMZ alone [13,14]. The effectiveness of radiotherapy will be discussed in detail in section 3.3.

\subsection{Optimizing radiotherapy}

Efforts have been made to investigate whether an adjusted radiotherapy method could further improve survival. A retrospective study applied dose-escalated radiotherapy (70 Gy/35) plus standard concomitant and adjuvant TMZ and proved that it significantly prolonged the OS and PFS of umMGMT patients. The median OS increased from 8 months to 14 months, and PFS increased from 5 months to 9 months. This study was the first to moderately escalate radiation specifically in patients with umMGMT and was confirmed effective [38]. A recent meta-analysis reported that dose-escalated radiotherapy exceeded the effect of standard radiotherapy alone [39]. However, when combined with temozolomide, the superiority was insignificant.

Dose-escalated radiotherapy was also coregistered with positron emission tomography (PET) to better delineate the target volume. A prospective phase II clinical trial utilized 3,4-dihydroxy-6-[18F]fluoro-L-phenylalanine (18F-DOPA) PET guided dose-escalated radiotherapy(51 60 76 Gy/30)[40]. In umMGMT patients, 6-months PFS (PFS-6) reached $79.5 \%$ (primary end-point set at 75\%) and PFS was 8.7 months compared to the historical control of 6.6 months.

Among all nGBM patients, elderly patients appear to rely more on radiotherapy, since elderly patients are likely to be more sensitive to the toxicity of intensive chemotherapy. The phase III NOA-08 trial suggested that the event-free survival (EFS) was longer in elderly umMGMT patients treated with radiotherapy alone than in patients treated with TMZ alone $\left(100 \mathrm{mg} / \mathrm{m}^{2}\right) \quad[14,41]$. And the OS of radiotherapy alone was no worse than TMZ alone. A meta-analysis suggested that radiotherapy alone was 
associated with a longer OS. Moreover, TMZ alone was associated with increased side effects in patients with umMGMT.

One of the purposes of hypofractionated radiotherapy is to reduce the number of hospital visits and avoid drop-out due to deterioration or disease progression. A phase III trial, the Nordic trial, compared TMZ alone, hypofractionated radiotherapy (34 Gy/10) and standard radiotherapy [13]. Patients generally responded better to TMZ alone and hypofractionated radiotherapy. The treatment effect of hypofractionated radiotherapy was independent of the MGMT methylation status. Therefore, for patients with umMGMT, hypofractionated radiotherapy might be considered prior to TMZ treatment. Another phase III trial used hypofractionated radiation (40 Gy/15) plus concomitant and adjuvant TMZ [37]. Hypofractionated radiotherapy plus TMZ was proven to be more effective than radiotherapy alone. The OS was 10.0 months compared with 7.9 months in umMGMT patients. This study illustrated that the addition of TMZ to radiation is, although less effective, still beneficial for elderly patients, even with a reduced overall radiation dose.

In summary, for adult umMGMT patients, radiotherapy plus concomitant and adjuvant TMZ was still more effective than radiotherapy alone. For patients older than 65 years of age, hypofractionated radiotherapy plus $\mathrm{TMZ}$ is an available choice. If one of the schedules must be eliminated from the plan, umMGMT patients should choose radiotherapy alone first, while mMGMT patients should consider otherwise.

\subsection{Promising clinical trials}

Despite all these improvements, the prognosis of umMGMT patients remains poor. Therefore, investigators have paved the way for clinical trials of novel or traditional chemotherapies and other therapies that could substitute or compensate for TMZ. However, the results were generally unsatisfying. Only a few treatments resulted in slight improvements. A list of clinical trials with both positive and negative results is summarized in Table 1.

\subsubsection{Tumor Treating Fields}

Tumor treating fields (TTF) is an external device that can eliminate tumors regardless of the MGMT methylation status. TTF utilize an electric field to interfere with the mitosis of tumor cells and thus inhibit tumor progression. In a randomized phase III clinical trial, EF-14, including 695 nGBM patients [42-44], TTF plus TMZ were administered after radiotherapy. The addition of TTF was associated with prolonged OS and PFS for both MMGMT and umMGMT subgroups. Among the 304 umMGMT patients, OS was prolonged from 14.7 months to 16.9 months. Quality of life was not affected. A pilot study reported the initial result of concurrent radiotherapy plus TTF, which is supposed to induce synergistic effect [45]. Toxicity was tolerable in this initial report.

Table 1. Outcomes of clinical trials for newly diagnosed GBM patients with unmethylated MGMT

\begin{tabular}{|c|c|c|c|c|c|c|c|}
\hline Trial & Phase & Year & Experiment arm & Control & Mechanism & Benefit & Patient subgroup \\
\hline EORTC-NCIC & 3 & 2005,2009 & $\mathrm{RT}+\mathrm{TMZ}$ & RT & & OS, PFS & \\
\hline NOA-08 & 3 & 2012,2020 & $\mathrm{RT}$ & CRT & & OS, EFS & Elderly \\
\hline EORTC-26062-22061 & 3 & 2017 & HF-RT(40Gy/15) +TMZ & HF-RT & RT & OS & Elderly \\
\hline Nordic & 3 & 2012 & HF-RT(34Gy/10) & RT or TMZ alone & RT & OS & Elderly \\
\hline NCT01991977 & 2 & 2021 & 18F-DOPA-PET+CRT & historical & $\mathrm{PET}+\mathrm{RT}$ & PFS & \\
\hline NCT00509821 & 2 & 2013 & $\mathrm{ENZ}+\mathrm{RT}$ & single arm & PKC & OS & \\
\hline ASPECT & 3 & 2013 & Adenovirus +RT (+TMZ) & RT (+TMZ) & Gene therapy & PFS & \\
\hline CORE & $1 / 2$ & $\begin{array}{l}2015 \\
2016\end{array}$ & $\mathrm{CIL}+\mathrm{CRT}$ & CRT & Integrin & OS, PFS & av $\beta 3$ integrin \\
\hline GLARIUS & 2 & $\begin{array}{l}2016 \\
2018\end{array}$ & $\mathrm{BEV}+\mathrm{IRI}+\mathrm{RT}$ & CRT & VEGF, topoisomerase & PFS6, PFS & \\
\hline ARTE & 2 & 2018 & BEV+HF- RT(40Gy/15) & HF-RT & VEGF, RT & PFS & \\
\hline EF-14 & 3 & 2017 & $\mathrm{TTF}+\mathrm{CRT}$ & CRT & TTF & OS, PFS & \\
\hline retrospective study & - & 2020 & valganciclovir +CRT & CRT & anti CMV & OS, PFS & \\
\hline EORTC 26082 & 2 & 2016 & TEM+RT & CRT & PI3K & insignificant & mTORser2448 \\
\hline OSAG 101-BSA-05 & 3 & 2015 & NIM+CRT & CRT & EGFR & OS & Akt, mTORC \\
\hline ExCentric & 2 & 2016 & $\mathrm{CIL}+\mathrm{PRO}+\mathrm{CRT}$ & single arm & Integrin, DNA-alkylating & insignificant & \\
\hline NCT00720356 & 2 & 2016 & $\mathrm{ERL}+\mathrm{BEV}+\mathrm{CRT}$ & single arm & EGFR, VEGF & insignificant & \\
\hline BrUOG 244 & 2 & 2018 & $\mathrm{PPX}+\mathrm{RT}$ & CRT & Paclitaxel & insignificant & \\
\hline NCT00998010 & 2 & 2018 & $\mathrm{BOR}+\mathrm{CRT}$ & single arm & Protease inhibitor & insignificant & \\
\hline VERTU & 2 & 2021 & veliparib+CRT & CRT & Protease inhibitor & PFS & \\
\hline CHECKMATE 498 & 3 & 2019 & NIV+RT & $\mathrm{N} / \mathrm{A}$ & PD-1 inhibitor & insignificant & \\
\hline
\end{tabular}

RT, radiotherapy; TMZ, temozolomide; CRT, standard chemoradiotherapy; HF, hypofractionated; OS, overall survival; PFS, progression free survival; EFS, event free survival; CIL, cilengitide; BEV, bevacizumab; TTF, tumor treating field; CMV, cytomegalovirus;TEM, temsirolimus; NIM, nimotuzumab; ENZ, enzastaurin; PRO, procarbazine; ERL, erlotinib; PPX, paclitaxel poliglumex; BOR, bortezomib. 
TTF appear to be a promising treatment method apart from the limitations of chemotherapy. It has been officially included in National Comprehensive Cancer Network (NCCN) guideline of CNS tumors. TTF is independent of known molecular biomarkers of GBM, and can combine swiftly with standard therapy. The hindrance of TTF is that its effectiveness relies heavily on the put-on time per day, which cannot be reached in some patients due to intolerable skin reaction or poor patient compliance.

\subsubsection{Chemotherapy}

Recently a phase II clinical trial recruited 47 nGBM patients with 31 umMGMT and applied the concomitant and adjuvant combination of temozolomide, vincristine and interferon [46]. A 2-year overall survival was $40.7 \%$ for the entire cohort, exceeding the historical control. PFS reached 11.0 months irrespective of MGMT methylation status. This study revealed a possible combination of chemotherapy drugs in addition to temozolomide. However, the increased adverse effect may limit the use of this study plan.

Dianhydrogalactitol (VAL-083) is another kind of DNA-alkylating agent independent of MGMT repair and temozolomide pathway. In the halfway report of this phase II clinical trial targeting umMGMT nGBM, 10 patients remained progression free, while another 12 patients progressed with PFS of 9.9 months [47]. It turns out that VAL-083 may be promising for umMGMT patients in substitute of temozolomide. However, this conclusion awaits further clinical results.

Paclitaxel poliglumex (PPX) is a polymer agent derived from the traditional chemotherapy agent paclitaxel. In phase II clinical trial (BrUOG 244) [48], PPX $\left(10 \mathrm{mg} / \mathrm{m}^{2}\right)$ was administered during the radiotherapy session on the first day of each week to enhance tumor sensitivity to radiotherapy. Adjuvant TMZ therapy was commenced after radiotherapy. However, this study showed no clinical benefit for umMGMT patients.

\subsubsection{Integrin inhibitors}

The significant efforts lie in targeted therapy. However, the study of cilengitide, an integrin inhibitor, has reached a dead end. Integrins are important proteins that mediate angiogenesis in nGBM and cell apoptosis. A previous phase I/II study of the CORE trial revealed that both standard and intensive cilengitide treatments were able to prolong the OS and PFS of umMGMT patients. Further analysis also showed that high av $\beta 3$ integrin expression in umMGMT nGBM was associated with prolonged PFS independent of the treatment type [49]. Nevertheless, in the subsequent phase III
CENTRIC EORTC 26071-22072 study that only included mMGMT patients, cilengitide $(2000 \mathrm{mg}$, twice a week) plus radiochemotherapy failed, with no survival benefit. Cilengitide has thereafter not been further studied as a treatment for GBM.

Another phase II trial specifically targeting umMGMT patients added procarbazine to cilengitide [50]. Procarbazine is a DNA alkylating agent that has been shown to inhibit MGMT independent of TMZ in preclinical trials. Concomitant and adjuvant TMZ was replaced with TMZ $\left(60 \mathrm{mg} / \mathrm{m}^{2}\right)$ plus procarbazine (50-100 mg) during and after radiotherapy. However, this combination of targeted treatments did not produce a positive outcome.

\subsubsection{Anti-angiogenesis therapy}

Another novel medication that has been extensively studied was bevacizumab, which resulted in prolonged PFS but not OS [51]. Bevacizumab is an antibody against vascular endothelial growth factor (VEGF). In the study published by Gilbert et al., bevacizumab improved PFS from 5.4 months to 9.8 months in umMGMT patients. Another phase III trial published by Chinot et al. [52] documented similar results that only PFS was improved. And more adverse events were associated with bevacizumab than with placebo. A phase II trial ARTE targeted elderly patients. Bevacizumab and hypofractionated radiotherapy (40 Gy/15) was compared with radiotherapy alone, showing prolonged PFS but no OS benefit in umMGMT patients [53].

The combination of bevacizumab and other medications did not improve OS either. In the phase II GLARIUS trial, TMZ was substituted by bevacizumab plus irinotecan [54]. The experimental arm showed an increase in the PFS-6 (from $42.6 \%$ to $79.3 \%$ ) and PFS (from 5.99 to 9.7 months), but not OS. In another phase II trial specifically targeting umMGMT patients [55], erlotinib plus bevacizumab therapy were commenced after radiotherapy plus concomitant TMZ therapy. This adjuvant therapy was well tolerated but did not improve PFS or OS.

Although studies of bevacizumab have all shown improvement in PFS but not OS, bevacizumab is well effective in reducing brain edema and improving symptoms, as a substitute of glucocorticoids, but without the extensive long-term adverse effects. It has also shown potential benefit to the immune microenvironment of GBM [56]. Therefore, bevacizumab is still an actively used agent in clinical practice.

\subsubsection{PKC/PI3K inhibitors}

Approaches to intervene in other oncogenic pathways have also been researched. In a phase II 
clinical trial [57], enzastaurin, a PKC inhibitor, was administered explicitly to umMGMT patients before, during and after radiotherapy. In this single-arm study, while the dose was safely tolerated, the primary endpoint PFS-6 was unfortunately not reached. For umMGMT patients who underwent partial or complete resection, the OS was 15.4 and 18.9 months, respectively. Nonetheless, PFS-6 is no longer used as the primary objective in many trials because of its limitations. Therefore, although failing to meet its primary endpoint, enzastaurin should be considered a promising agent for umMGMT patients.

\subsection{6 mTOR inhibitor}

In the phase II clinical trial EORTC 26082 [58], radiotherapy plus concomitant and maintenance temsirolimus, an mTOR inhibitor, was administered at a dose of $25 \mathrm{mg}$ per week. Neither OS nor PFS was significantly improved for umMGMT patients. A further subgroup analysis showed that a particular group of mTOR (Ser2448)-positive patients may benefit from radiotherapy plus temsirolimus treatment. Further clinical evidence is needed to confirm this finding.

\subsubsection{Protease inhibitors}

In addition to traditional chemotherapy, protease inhibitors have also been considered. However, a phase II clinical trial declared that the umMGMT subgroup had a relatively poor prognosis after treatment with standard radiochemotherapy plus bortezomib $\left(1.3 \mathrm{mg} / \mathrm{m}^{2}\right.$ on days $1,4,8$, and 11 of the 28-day cycle) [59]. Although this trial recruited only 23 patients (only 13 umMGMT patients), the overtly poor outcome was unlikely to be reversed with the inclusion of more patients.

Veliparib is a poly ADP-ribose polymerase (PARP) inhibitor that kills tumor cells and sensitizes radiotherapy in preclinical studies [60]. The phase II clinical trial VERTU, particularly targeting umMGMT patients, added concomitant and adjuvant veliparib to standard radiochemotherapy [61]. PFS-6 achieved $46 \%$ over $31 \%$ in experiment arm and control, respectively. However, OS was not prolonged (12.7 vs. 12.8 months).

\subsubsection{Anti-EGFR antibodies}

Likewise, nimotuzumab, an EGFR antibody, was a beneficial treatment with predictive biomarkers from the Akt and mTORC signalling pathways. In an open-label phase III trial [62], nimotuzumab was added to standard TMZ therapy. For patients with umMGMT and EGFR amplification, PFS increased from 5.8 to 8.3 months, while OS from 15.5 to 19.5 months. A further subgroup analysis was conducted [63]. Akt and mTORC, which serve as downstream effectors of EGFR inhibition, have been defined as positive predictive biomarkers for nimotuzumab treatment. However, most patients do not experience this potential benefit, and for those included in the study, the outcome was not as promising as that observed in mMGMT patients.

\subsubsection{Immunotherapy}

DCVax-L was a phase III clinical trial that utilized the autologous tumor lysate-pulsed dendritic cell (DC) vaccine to treat nGBM patients[64]. DCVax-L was administered after surgery and radiotherapy, with a dose of 2.5 million DC intradermally. For the umMGMT group, OS was 19.8 months from surgery. The limitation of this study is that patients with symptoms of early progression were excluded from the study cohort. Therefore, selection bias may have contributed to the good OS outcome.

Recently, the checkpoint inhibitor nivolumab was also reported to have failed in a phase III trial in umMGMT (Checkmate 498) and mMGMT (Checkmate 548) patients. Nivolumab was added as an adjuvant to standard radiochemotherapy. The primary endpoint, OS, was not reached by the time of the announcement, although toxicity was well tolerated. On the other hand, the use of a neoadjuvant PD-1 inhibitor in GBM may be promising. In a phase II clinical trial analyzing neoadjuvant PD-1 in 27 rGBM and 3 nGBM patients, increased immune activity was detected [65]. Two of the three nGBM patients, both of whom were mMGMT, survived for over 28 and 33 months, respectively. The efficacy and effectiveness of a neoadjuvant PD-1 inhibitor await future investigation.

\subsubsection{Virus-related treatment}

According to previous studies, patients with GBM are often diagnosed with cytomegalovirus infection, potentially a treatment target. In the phase III clinical trial ASPECT, adenovirus-mediated gene therapy called sitimagene ceradenovec was commenced to treat nGBM patients [66]. This genetically altered adenovirus carrying a prodrug converting enzyme for ganciclovir was directly injected into the tumor cavity, followed by intravenous ganciclovir, radiotherapy with or without TMZ. In the umMGMT subgroup, patients had prolonged time to death or re-intervention, regardless of TMZ use. Patients with umMGMT experienced a greater benefit than mMGMT patients.

A retrospective study of GBM patients receiving valganciclovir in addition to standard radiochemotherapy has yielded positive results for umMGMT patients. Valganciclovir exerts an anti-cytomegalo- 
virus effect and has significantly improved the OS for umMGMT patients from 11.6 months to 21.1 months. Although this retrospective study only included some eligible patients for the MGMT methylation analysis, valganciclovir represents a promising treatment option and future direction.

The first oncolytic virus therapy in GBM has been approved by the Japan Ministry of Health, Labour and Welfare in 2021. Teserpaturev (Delytact/ G47) is a herpes simplex virus 1 (HSV-1) oncolytic virus that has been genetically modified to selectively replicate in cancer cells [67]. The correlated phase II clinical trial outcome has not been published yet.

\subsection{Recurrent glioblastoma}

Unlike nGBM, a well-defined standard therapy is not available for rGBM patients. Strategies, such as a second resection, re-irradiation, bevacizumab, lomustine and TMZ rechallenge, are generally considered beneficial.

A second resection should only be performed when a survival benefit will be obtained. A retrospective study has found that rGBM patients who underwent GTR and STR of the tumor experienced longer OS than patients with biopsy or without surgery [68]. For umMGMT rGBM patients, the superiority of GTR over STR was also noted. Another study, however, suggested a more conservative attitude toward a second resection. In the post hoc analysis of the cohort in the DIRECTOR trial, patients experienced prolonged survival only if GTR of the advancing tumor can still be achieved at the first recurrence [69]. Therefore, the choice of a second resection at recurrence should be carefully evaluated for individuals considering the personal needs, functional status, tumor size and location, preferably only if GTR is possible.

At the time of relapse, the MGMT promoter methylation status is changed bidirectionally in a range of 8-37\% GBM patients due to selective pressure and intratumor heterogeneity [70]. More patients lost MGMT methylation than gained. This causes complications and opportunities for TMZ rechallenge. In both intensive or low dose TMZ rechallenge [71,72], mMGMT patients showed significantly longer PFS than umMGMT patients. The phase II GICNO trial [73] suggested that TMZ $\left(75 \mathrm{mg} / \mathrm{m}^{2} / \mathrm{d}\right)$ was able to increase the PFS-6 for rGBM patients regardless of MGMT status. Though other studies still regarded MGMT methylation as a predictive biomarker of rGBM

In addition to TMZ rechallenge, antiangiogenic therapy is one of the most commonly used therapies for treating rGBM patients. For umMGMT patients, one promising treatment combines onartuzumab, an
anti-MET antibody, with bevacizumab [74]. The phase II clinical trial evaluated onartuzumab $(15 \mathrm{mg} / \mathrm{kg})$ plus bevacizumab $(15 \mathrm{mg} / \mathrm{kg})$ and showed a negative effect on the overall cohort, especially mMGMT patients. However, in the umMGMT subgroup, the results indicated prolonged survival and slower progression, suggesting that onartuzumab plus bevacizumab might be a promising treatment for umMGMT patients. Further clinical evidence targeting umMGMT patients is needed.

Table 2. Ongoing phase II/III clinical trials specifically for umMGMT patients or with umMGMT subgroup

\begin{tabular}{|c|c|c|c|c|}
\hline & Experiment arm & Mechanism & Phase & Trial \\
\hline 1 & $\begin{array}{l}\text { Disulfiram+Copper } \\
\text { Gluconate+CRT }\end{array}$ & Chemotherapy & 2 & NCT03363659 \\
\hline 2 & $\begin{array}{l}\text { Nivolumab+Ipilimumab+ } \\
\text { short-course RT }\end{array}$ & $\begin{array}{l}\text { PD-1 inhibitor } \\
\text { CTLA-4 inhibitor }\end{array}$ & 2 & NCT03367715 \\
\hline 3 & $\begin{array}{l}\text { Nivolumab+Ipilimumab+R } \\
T\end{array}$ & $\begin{array}{l}\text { PD-1 inhibitor } \\
\text { CTLA-4 inhibitor }\end{array}$ & $2 / 3$ & $\begin{array}{l}\text { NCT03367715 } \\
\text { NCT04396860 }\end{array}$ \\
\hline 4 & $\begin{array}{l}\text { CMV pp65 DC vaccine } \\
+ \text { CRT }\end{array}$ & Immune vaccine & 2 & $\begin{array}{l}\text { I-ATTAC } \\
\text { NCT03927222 }\end{array}$ \\
\hline 5 & $\begin{array}{l}\text { CMV pp65 DC vaccine+ } \\
\text { varlilumab+CRT }\end{array}$ & $\begin{array}{l}\text { Immune vaccine } \\
\text { anti-CD27 }\end{array}$ & 2 & NCT03688178 \\
\hline 6 & Paxalisib+CRT & PI3K/mTOR inhibitor & 2 & NCT03522298 \\
\hline 7 & Dianhydrogalactitol+CRT & Chemotherapy & 2 & $\begin{array}{l}\text { NCT02717962 } \\
\text { NCT03050736 }\end{array}$ \\
\hline 8 & Olaptesed Pegol+RT & CXCL-12 inhibitor & $1 / 2$ & $\begin{array}{l}\text { GLORIA } \\
\text { NCT04121455 }\end{array}$ \\
\hline 9 & Bortezomib+RT+TMZ & Protease inhibitor & $1 / 2$ & $\begin{array}{l}\text { BORTEM-17 } \\
\text { NCT03643549 }\end{array}$ \\
\hline 10 & Temferon+RT & $\begin{array}{l}\text { Lentivirus } \\
\text { CD34+enriched HSPC }\end{array}$ & $1 / 2$ & $\begin{array}{l}\text { TEM-GBM } \\
\text { NCT03866109 }\end{array}$ \\
\hline 11 & $\begin{array}{l}\text { Pembrolizumab+CRT } \\
\text { Pembrolizumab+HSPPC-96 } \\
\text { +CRT }\end{array}$ & $\begin{array}{l}\text { PD-1 inhibitor } \\
\text { Immune vaccine }\end{array}$ & 2 & NCT03018288 \\
\hline 12 & $\begin{array}{l}\text { INO-5401+INO-9012+ } \\
\text { cemiplima+CRT }\end{array}$ & $\begin{array}{l}\text { DNA plasmid } \\
\text { PD-1 inhibitor }\end{array}$ & $1 / 2$ & NCT03491683 \\
\hline 13 & $\begin{array}{l}\text { APG101, Alectinib, } \\
\text { Idasanutlin, Atezolizumab, } \\
\text { Vismodegib, Palbociclib, } \\
\text { Temsirolimus }\end{array}$ & Targeted therapy & $1 / 2$ & $\begin{array}{l}\mathrm{N}^{2} \mathrm{M}^{2}(\mathrm{NOA}-20) \\
\text { NCT03158389 }\end{array}$ \\
\hline 14 & Durvalumab $+\mathrm{RT}$ & PD-L1 inhibitor & 2 & NCT02336165 \\
\hline 15 & $\begin{array}{l}\text { Pamiparib+RT, } \\
\text { Pamiparib+CRT }\end{array}$ & PARP $1 / 2$ inhibitor & $1 / 2$ & NCT03150862 \\
\hline 16 & Sunitinib+CRT & TKI & 2 & NCT02928575 \\
\hline 17 & Chlorpromazine $+\mathrm{CRT}$ & $\begin{array}{l}\text { dopamine receptor } \mathrm{D} 2 \\
\text { antagonist }\end{array}$ & 2 & NCT04224441 \\
\hline 18 & Selinexor+RT & $\begin{array}{l}\text { selective inhibitor of } \\
\text { nuclear export }\end{array}$ & 2 & NCT04421378 \\
\hline 19 & Apatinib+TMZ & TKI & 2 & $\begin{array}{l}\text { ChiCTR190002 } \\
0561\end{array}$ \\
\hline 20 & Anlotinib+CRT & TKI & 2 & NCT04725214 \\
\hline
\end{tabular}

RT, radiotherapy; CRT, standard chemoradiotherapy; TMZ, temozolomide; HSPC, Hydrogenated Soybean Phospholipids; TKI, tyrosine kinase inhibitor.

\subsection{Future directions}

Ongoing phase II or phase III clinical trials either specifically designed for patients with umMGMT or a cohort of umMGMT patients are listed in Table 2, including studies registered at clinicaltrials.gov, clinicaltrialsregister.eu, anzctr.org.au and chictr.org.cn. Two phase III trials were identified. Both of them were related to immune therapy. Several molecules, including immune checkpoint inhibitors, dendritic cells, protease inhibitors, PARP inhibitors, 
and mTOR inhibitors, are being investigated.

In the future, precision medicine may lead to higher therapeutic effectiveness. In a phase $\mathrm{I} / \mathrm{Ib}$ clinical trial published in Nature in 2019, a personalized neoantigen vaccine was shown to trigger the immune response mediated by neoantigenspecific $\mathrm{T}$ cells in umMGMT GBM patients [75]. A neoepitope-related peptide was designed specifically for each patient. This vaccine was able to trigger the $T$ cell-mediated immune response and alter the immune environment of GBM. Further studies are needed to verify its effectiveness.

Another study design was published based on the idea of precision medicine. The phase I/II NOA-20 trial intended to match patients to the molecular subgroups [76]. After the molecular analysis, the patients who possessed an ALK fusion, CDK4/6 amplification, mTOR phosphorylation, MDM2 amplification or SHH amplification were assigned to receive different targeted medications separately. Patients without those markers were randomized to use TMZ, asinercept (APG101) or the checkpoint inhibitor. This study design was based on sophisticated bioinformatics analyses and was believed to be more efficient for clinical trials.

\section{Conclusions}

The umMGMT status is a lynchpin factor that leads to TMZ resistance in GBM patients. However, only $40 \%$ of GBM patients carry hypermethylated MGMT, leaving $60 \%$ of patients with minimal benefits. In addition to surgical resection and standard TMZ radiochemotherapy, bevacizumab plus irinotecan, enzastaurin plus TMZ and TTF also benefit umMGMT patients to a certain extent. For elderly patients with umMGMT, hypofractionated radiotherapy plus $\mathrm{TMZ}$ is preferred when tolerable. If not, standard or hypofractionated radiotherapy alone should be considered superior to TMZ alone. For umMGMT GBM patients, recent clinical trials have failed to yield promising outcomes. Researchers have been studying the ways to reverse TMZ resistance or to overpass MGMT pathways. The success of TTF was an example of therapy or even external device with novel anti-tumor mechanisms. Because of the high inter- and intra-tumor heterogeneity and dynamic transformation of MGMT methylation status, precision medicine is currently of particular therapeutic value.

\section{Acknowledgements}

We thank American Journal Experts for language editing.

\section{Financial Support}

This work was supported by the Chinese Academy of Medical Sciences Innovation Fund for Medical Sciences [grant number: 2016-I2M2-001], the Beijing Municipal Natural Science Foundation [grant number: 7202150 and 19JCZDJC64200(Z)], and the Tsinghua University-Peking Union Medical College Hospital Initiative Scientific Research Program [grant number: 2019ZLH101].

\section{Author Contributions}

Dr. Delin Liu drafted and organized the manuscript of the review. Dr. Tianrui Yang drew the figure and revised part of the article about immune therapy. Dr. Wenbin Ma supervised the review, provided essential advice for content and organization of the article. Dr. Yu Wang generally supervised and organized the article and provided insights of the future direction. All authors agree to the content of the manuscript.

\section{Competing Interests}

The authors have declared that no competing interest exists.

\section{References}

1. Miller KD, Ostrom QT, Kruchko C, et al. Brain and other central nervous system tumor statistics, 2021. CA Cancer J Clin. 2021; 13:caac. 21693.

2. Stupp R, Mason WP, van den Bent MJ, et al. Radiotherapy plus Concomitant and Adjuvant Temozolomide for Glioblastoma. N Engl J Med. 2005; 352:987-996.

3. Hegi ME, Diserens A-CC, Gorlia T, et al. MGMT Gene Silencing and Benefit from Temozolomide in Glioblastoma. N Engl J Med. 2005; 352:997-1003.

4. Jiang T, Mao Y, Ma W, et al. CGCG clinical practice guidelines for the management of adult diffuse gliomas. Cancer Lett. 2016; 375:263-273.

5. Ostrom OT, Cioffi G, Gittleman H, et al. CBTRUS Statistical Report: Primary Brain and Other Central Nervous System Tumors Diagnosed in the United States in 2012-2016. Neuro Oncol. 2019; 21:V1-V100.

6. Tolcher AW, Gerson SL, Denis L, et al. Marked inactivation of O6-alkylguanine-DNA alkyltransferase activity with protracted temozolomide schedules. Br J Cancer. 2003; 88:1004-1011.

7. Fan $\mathrm{CH}$, Liu WL, Cao H, et al. O6-methylguanine DNA methyltransferase as a promising target for the treatment of temozolomide-resistant gliomas. Cell Death Dis. 2013; 4:e876-8

8. Quiros S, Roos WP, Kaina B. Processing of O 6 -methylguanine into DNA double-strand breaks requires two rounds of replication whereas apoptosis is also induced in subsequent cell cycles. Cell Cycle. 2010; 9:168-178.

9. Pegg AE, Dolan ME, Moschel RC. Structure, Function, and Inhibition of O6-Alkylguanine-DNA Alkyltransferase. Prog Nucleic Acid Res Mol Biol. 1995; 51:167-223.

10. Hegi ME, Liu L, Herman JG, et al. Correlation of O6-methylguanine methyltransferase (MGMT) promoter methylation with clinical outcomes in glioblastoma and clinical strategies to modulate MGMT activity. J Clin Oncol. 2008; 26:4189-4199.

11. Sarkaria JN, Kitange GJ, James CD, et al. Mechanisms of chemoresistance to alkylating agents in malignant glioma. Clin Cancer Res. 2008; 14:2900-2908.

12. Stupp R, Hegi ME, Mason WP, et al. Effects of radiotherapy with concomitant and adjuvant temozolomide versus radiotherapy alone on survival in glioblastoma in a randomised phase III study: 5-year analysis of the EORTC-NCIC trial. Lancet Oncol. 2009; 10:459-466.

13. Malmström A, Grønberg BH, Marosi C, et al. Temozolomide versus standard 6-week radiotherapy versus hypofractionated radiotherapy in patients older than 60 years with glioblastoma: The Nordic randomised, phase 3 trial. Lancet Oncol. 2012; 13:916-926.

14. Wick W, Platten M, Meisner C, et al. Temozolomide chemotherapy alone versus radiotherapy alone for malignant astrocytoma in the elderly: The NOA-08 randomised, phase 3 trial. Lancet Oncol. 2012; 13:707-715.

15. Alnahhas I, Alsawas M, Rayi A, et al. Characterizing benefit from temozolomide in MGMT promoter unmethylated and methylated 
glioblastoma: a systematic review and meta-analysis. Neuro-Oncology Adv. 2020; $2: 1-7$.

16. Cohen KJ, Pollack IF, Zhou T, et al. Temozolomide in the treatment of high-grade gliomas in children: a report from the Children's Oncology Group. Neuro Oncol. 2011; 13:317-323.

17. Louis DN, Perry A, Wesseling P, et al. The 2021 WHO Classification of Tumors of the Central Nervous System: a summary. Neuro Oncol. 2021; 23:1231-1251.

18. Bady P, Sciuscio D, Diserens AC, et al. MGMT methylation analysis of glioblastoma on the Infinium methylation BeadChip identifies two distinct CpG regions associated with gene silencing and outcome, yielding a prediction model for comparisons across datasets, tumor grades, and CIMP-status. Acta Neuropathol. 2012; 124:547-560.

19. Karayan-Tapon L, Quillien V, Guilhot J, et al. Prognostic value of O6-methylguanine-DNA methyltransferase status in glioblastoma patients, assessed by five different methods. J Neurooncol. 2010; 97:311-322.

20. Quillien V, Lavenu A, Karayan-Tapon L, et al. Comparative assessment of 5 methods (methylation-specific polymerase chain reaction, methylight, pyrosequencing, methylation-sensitive high-resolution melting, and immunohistochemistry) to analyze O6-methylguanine-DNAmethyltranferase in a series of 100 . Cancer. 2012; 118:4201-4211.

21. Johannessen LE, Brandal P, Myklebust TA, et al. MGMT gene promoter methylation status - Assessment of two pyrosequencing kits and three methylation-specific PCR methods for their predictive capacity in glioblastomas. Cancer Genomics and Proteomics. 2018; 15:437-446.

22. Lattanzio L, Borgognone M, Mocellini C, et al. MGMT promoter methylation and glioblastoma: A comparison of analytical methods and of tumor specimens. Int J Biol Markers. 2015; 30:e208-e216.

23. Hegi ME, Genbrugge E, Gorlia T, et al. MGMT Promoter Methylation Cutoff with Safety Margin for Selecting Glioblastoma Patients into Trials Omitting Temozolomide: A Pooled Analysis of Four Clinical Trials. Clin Cancer Res. 2018; 25:1809-1816.

24. Estival A, Sanz C, Ramirez JL, et al. Pyrosequencing versus methylationspecific PCR for assessment of MGMT methylation in tumor and blood samples of glioblastoma patients. Sci Rep. 2019; 9:11125.

25. Moon WJ, Choi JW, Roh HG, et al. Imaging parameters of high grade gliomas in relation to the MGMT promoter methylation status: The CT, diffusion tensor imaging, and perfusion MR imaging. Neuroradiology. 2012; 54:555-563.

26. Kanazawa $T$, Minami $Y$, Jinzaki $M$, et al. Predictive markers for MGMT promoter methylation in glioblastomas. Neurosurg Rev. 2019; 42:867-876.

27. Binabaj MM, Bahrami A, ShahidSales S, et al. The prognostic value of MGMT promoter methylation in glioblastoma: A meta-analysis of clinical trials. J Cell Physiol. 2018; 233:378-386.

28. Everhard S, Kaloshi G, Crinière E, et al. MGMT methylation: A marker of response to temozolomide in low-grade gliomas. Ann Neurol. 2006; 60:740743

29. Taylor JW, Schiff D. Treatment Considerations for MGMT-Unmethylated Glioblastoma. Curr Neurol Neurosci Rep. 2015; 15:507.

30. Kitange GJ, Carlson BL, Schroeder MA, et al. Induction of MGMT expression is associated with temozolomide resistance in glioblastoma xenografts. Neuro Oncol. 2008; 11:281-291.

31. Katsigiannis S, Grau S, Krischek B, et al. MGMT-Positive vs MGMT-Negative Patients with Glioblastoma: Identification of Prognostic Factors and Resection Threshold. Neurosurgery. 2021; 88:E323-E329.

32. Sales AHA, Bette S, Barz M, et al. Role of postoperative tumor volume in patients with MGMT-unmethylated glioblastoma. J Neurooncol. 2019; 142:529-536.

33. Rennert RC, Khan U, Bartek J, et al. Laser ablation of abnormal neurological tissue using robotic neuroblate system (laantern): Procedural safety and hospitalization. Neurosurgery. 2020; 86:538-547.

34. Kamath AA, Friedman DD, Akbari SHA, et al. Glioblastoma Treated With Magnetic Resonance Imaging-Guided Laser Interstitial Thermal Therapy: Safety, Efficacy, and Outcomes. Neurosurgery. 2019; 84:836-843.

35. Bastos DC de A, Weinberg J, Kumar VA, et al. Laser Interstitial Thermal Therapy in the treatment of brain metastases and radiation necrosis. Cancer Lett. 2020; 489:9-18.

36. Di L, Wang $\mathrm{CP}$, Shah AH, et al. A Cohort Study on Prognostic Factors for Laser Interstitial Thermal Therapy Success in Newly Diagnosed Glioblastoma. Neurosurgery. 2021; 0:1-8.

37. Perry JR, Laperriere N, O'Callaghan CJ, et al. Short-Course Radiation plus Temozolomide in Elderly Patients with Glioblastoma. N Engl J Med. 2017; 376:1027-1037.

38. Tini $\mathrm{P}$, Nardone $\mathrm{V}$, Pastina $\mathrm{P}$, et al. Patients Affected by Unmethylated O(6)-Methylguanine-DNA Methyltransferase Glioblastoma Undergoing Radiochemotherapy May Benefit from Moderately Dose-Escalated Radiotherapy. Biomed Res Int. 2017; 2017:1-7.

39. Singh R, Lehrer EJ, Wang $M$, et al. Dose Escalated Radiation Therapy for Glioblastoma Multiforme: An International Systematic Review and Meta-Analysis of 22 Prospective Trials. Int J Radiat Oncol Biol Phys. 2021; 111:371-384

40. Laack NN, Pafundi D, Anderson SK, et al. Initial Results of a Phase 2 Trial of 18F-DOPA PET-Guided Dose-Escalated Radiation Therapy for Glioblastoma. Int J Radiat Oncol. 2021; 110:1383-1395.

41. Wick A, Kessler T, Platten M, et al Superiority of temozolomide over radiotherapy for elderly patients with RTK II methylation class, MGMT promoter methylated malignant astrocytoma. Neuro Oncol. 2020; 22:11621172

42. Stupp R, Taillibert S, Kanner AA, et al. Maintenance therapy with tumorTreating fields plus temozolomide vs temozolomide alone for glioblastoma a randomized clinical trial. JAMA - J Am Med Assoc. 2015; 314:2535-2543.

43. Stupp R, Taillibert S, Kanner A, et al. Effect of tumor-treating fields plus maintenance temozolomide vs maintenance temozolomide alone on survival in patients with glioblastoma a randomized clinical trial. JAMA - J Am Med Assoc. 2017; 318:2306-2316

44. Taphoorn MJB, Dirven L, Kanner AA, et al. Influence of treatment with tumor-treating fields on health-related quality of life of patients with newly diagnosed glioblastoma a secondary analysis of a randomized clinical trial. JAMA Oncol. 2018; 4:495-504.

45. Song A, Bar-Ad V, Martinez N, et al. Initial experience with scalp sparing radiation with concurrent temozolomide and tumor treatment fields (SPARE) for patients with newly diagnosed glioblastoma. J Neurooncol. 2020; 147:653661.

46. Asano K, Fumoto T, Matsuzaka M, et al. Combination chemoradiotherapy with temozolomide, vincristine, and interferon- $\beta$ might improve outcomes regardless of O6-methyl-guanine-DNA-methyltransferase (MGMT) promoter methylation status in newly glioblastoma. BMC Cancer. 2021; 21:1-12.

47. Guo C, Yang Q, Li J, et al. Phase 2 clinical trial of VAL-083 as first-line treatment in newly-diagnosed MGMT-unmethylated glioblastoma multiforme (GBM): Halfway report. Glioma. 2019; 2:167.

48. Elinzano H, Glantz M, Mrugala M, et al. PPX and Concurrent Radiation for Newly Diagnosed Glioblastoma Without MGMT Methylation. Am J Clin Oncol. 2018; 41:159-162.

49. Weller M, Nabors LB, Gorlia $\mathrm{T}$, et al. Cilengitide in newly diagnosed glioblastoma: biomarker expression and outcome. Oncotarget. 2016; 7:15018-15032.

50. Khasraw $M$, Lee $A$, Mccowatt $S$, et al Cilengitide with metronomic temozolomide, procarbazine, and standard radiotherapy in patients with glioblastoma and unmethylated MGMT gene promoter in ExCentric, an open-label phase II trial. J Neurooncol. 2016; 128:163-171.

51. Gilbert MR, Dignam JJ, Armstrong TS, et al. A Randomized Trial of Bevacizumab for Newly Diagnosed Glioblastoma. N Engl J Med. 2014; 370:699-708.

52. Chinot OL, Wick W, Mason W, et al. Bevacizumab plus RadiotherapyTemozolomide for Newly Diagnosed Glioblastoma. N Engl J Med. 2014; 370:709-722.

53. Wirsching HG, Tabatabai G, Roelcke U, et al. Bevacizumab plus hypofractionated radiotherapy versus radiotherapy alone in elderly patients with glioblastoma: The randomized, open-label, phase II ARTE trial. Ann Oncol. 2018; 29:1423-1430.

54. Herrlinger U, Schäfer N, Steinbach JP, et al. Bevacizumab Plus irinotecan versus temozolomide in newly diagnosed O6-methylguanine-DNA methyltransferase nonmethylated glioblastoma: The randomized GLARIUS trial. J Clin Oncol. 2016; 34:1611-1619.

55. Raizer JJ, Giglio P, Hu J, et al. A phase II study of bevacizumab and erlotinib after radiation and temozolomide in MGMT unmethylated GBM patients. J Neurooncol. 2016; 126:185-192.

56. Huinen ZR, Huijbers EJM, van Beijnum JR, et al. Anti-angiogenic agents overcoming tumour endothelial cell anergy and improving immunotherapy outcomes. Nat Rev Clin Oncol. 2021; 18:527-540.

57. Wick W, Steinbach JP, Platten $\mathrm{M}$, et al. Enzastaurin before and concomitant with radiation therapy, followed by enzastaurin maintenance therapy, in patients with newly diagnosed glioblastoma without MGMT promoter hypermethylation. Neuro Oncol. 2013; 15:1405-1412.

58. Wick W, Gorlia T, Bady P, et al. Phase II study of radiotherapy and temsirolimus versus radiochemotherapy with temozolomide in patients with newly diagnosed glioblastoma without MGMT promoter hypermethylation (EORTC 26082). Clin Cancer Res. 2016; 22:4797-4806.

59. Kong X-T, Nguyen NT, Choi YJ, et al. Phase 2 Study of Bortezomib Combined With Temozolomide and Regional Radiation Therapy for Upfront Treatment of Patients With Newly Diagnosed Glioblastoma Multiforme: Safety and Efficacy Assessment. Int J Radiat Oncol Biol Phys. 2018; 100:1195-1203.

60. Jue TR, Nozue K, Lester AJ, et al. Veliparib in combination with radiotherapy for the treatment of MGMT unmethylated glioblastoma. J Transl Med. 2017; 15:1-8.

61. Sim H-W, McDonald KL, Lwin Z, et al. A randomized phase II trial of veliparib, radiotherapy, and temozolomide in patients with unmethylated MGMT glioblastoma: the VERTU study. Neuro Oncol. 2021; 1-40.

62. Westphal M, Heese O, Steinbach JP, et al. A randomised, open label phase III trial with nimotuzumab, an anti-epidermal growth factor receptor monoclonal antibody in the treatment of newly diagnosed adult glioblastoma. Eur J Cancer. 2015; 51:522-532.

63. Ronellenfitsch MW, Zeiner PS, Mittelbronn M, et al. Akt and mTORC1 signaling as predictive biomarkers for the EGFR antibody nimotuzumab in glioblastoma. Acta Neuropathol Commun. 2018; 6:81.

64. Liau LM, Ashkan K, Tran DD, et al. First results on survival from a large Phase 3 clinical trial of an autologous dendritic cell vaccine in newly diagnosed glioblastoma. J Transl Med. 2018; 16:1.

65. Schalper KA, Rodriguez-Ruiz ME, Diez-Valle R, et al. Neoadjuvant nivolumab modifies the tumor immune microenvironment in resectable glioblastoma. Nat Med. 2019; 25:470-476 
66. Westphal M, Ylä-Herttuala S, Martin J, et al. Adenovirus-mediated gene therapy with sitimagene ceradenovec followed by intravenous ganciclovir for patients with operable high-grade glioma (ASPECT): a randomised, open-label, phase 3 trial. Lancet Oncol. 2013; 14:823-833.

67. Daiichi Sankyo. Press Release DELYTACT $®$ Oncolytic Virus G47 $\triangle$ Approved in Japan for Treatment of Patients with Malignant Glioma. 2021; .

68. Pala A, Schmitz AL, Knoll A, et al. Is MGMT promoter methylation to be considered in the decision making for recurrent surgery in glioblastoma patients? Clin Neurol Neurosurg. 2018; 167:6-10.

69. Suchorska B, Weller M, Tabatabai G, et al. Complete resection of contrast-enhancing tumor volume is associated with improved survival in recurrent glioblastoma - results from the DIRECTOR trial. Neuro Oncol. 2016; 18:549-556.

70. Feldheim J, Kessler AF, Monoranu CM, et al. Changes of O6-Methylguanine DNA Methyltransferase (MGMT) Promoter Methylation in Glioblastoma Relapse-A Meta-Analysis Type Literature Review. Cancers (Basel). 2019; 11:1837.

71. Weller M, Tabatabai G, Kästner B, et al. MGMT promoter methylation is a strong prognostic biomarker for benefit from dose-intensified temozolomide rechallenge in progressive Glioblastoma: The DIRECTOR Trial. Clin Cancer Res. 2015; 21:2057-2064.

72. Kim C, Kim HS, Shim WH, et al. Recurrent Glioblastoma: Combination of High Cerebral Blood Flow with MGMT Promoter Methylation Is Associated with Benefit from Low-Dose Temozolomide Rechallenge at First Recurrence. Radiology. 2016; 282:212-221.

73. Brandes AA, Tosoni A, Cavallo G, et al. Temozolomide 3 weeks on and 1 week off as first-line therapy for recurrent glioblastoma: Phase II study from Gruppo Italiano Cooperativo di Neuro-Oncologia (GICNO). Br J Cancer. 2006; 95:11551160.

74. Cloughesy T, Finocchiaro G, Belda-Iniesta C, et al. Randomized, double-blind, placebo-controlled, multicenter phase II study of onartuzumab plus bevacizumab versus placebo plus bevacizumab in patients with recurrent glioblastoma: Efficacy, safety, and hepatocyte growth factor and O6-methylguanine-DNA methy. J Clin Oncol. 2017; 35:343-351.

75. Keskin DB, Anandappa AJ, Sun J, et al. Neoantigen vaccine generates intratumoral $\mathrm{T}$ cell responses in phase Ib glioblastoma trial. Nature. 2019; 565:234-239.

76. Wick W, Dettmer S, Berberich A, et al. N 2 M 2 (NOA-20) phase I/II trial of molecularly matched targeted therapies plus radiotherapy in patients with newly diagnosed non-MGMT hypermethylated glioblastoma. Neuro Oncol. 2019; 21:95-105. 